\title{
SUFFICIENT CONDITIONS FOR MAXIMALLY EDGE-CONNECTED AND SUPER-EDGE-CONNECTED GRAPHS DEPENDING ON THE CLIQUE NUMBER
}

\author{
Lutz Volkmann \\ Lehrstuhl II für Mathematik \\ RWTH Aachen University \\ 52056 Aachen, Germany \\ e-mail: volkm@math2.rwth-aachen.de
}

\begin{abstract}
Let $G$ be a connected graph with minimum degree $\delta$ and edge-connectivity $\lambda$. A graph is maximally edge-connected if $\lambda=\delta$, and it is super-edgeconnected if every minimum edge-cut is trivial; that is, if every minimum edge-cut consists of edges incident with a vertex of minimum degree. The clique number $\omega(G)$ of a graph $G$ is the maximum cardinality of a complete subgraph of $G$. In this paper, we show that a connected graph $G$ with clique number $\omega(G) \leq r$ is maximally edge-connected or super-edge-connected if the number of edges is large enough. These are generalizations of corresponding results for triangle-free graphs by Volkmann and Hong in 2017.
\end{abstract}

Keywords: edge-connectivity, clique number, maximally edge-connected graphs, super-edge-connected graphs.

2010 Mathematics Subject Classification: 05C40.

\section{REFERENCES}

[1] D. Bauer, C. Suffel, F. Boesch and R. Tindell, Connectivity extremal problems and the design of reliable probabilistic networks, in: The Theory and Applications of Graphs, Kalamazoo MI (Wiley, New York, 1981) 45-54.

[2] G. Chartrand, A graph-theoretic approach to a communications problem, SIAM J. Appl. Math. 14 (1966) 778-781.

doi:10.1137/0114065

[3] A. Hellwig and L. Volkmann, Maximally edge-connected and vertex-connected graphs and digraphs: A survey, Discrete Math. 308 (2008) 3265-3296.

doi:10.1016/j.disc.2007.06.035 
[4] A.K. Kelmans Asymptotic formulas for the probability of $k$-connectedness of random graphs, Theory Probab. Appl. 17 (1972) 243-254. doi:10.1137/1117029

[5] P. Turán, On an extremal problem in graph theory, Mat. Fiz. Lapok 48 (1941) 436-452, in Hungarian.

[6] L. Volkmann and Z.-M. Hong, Sufficient conditions for maximally edge-connected and super-edge-connected graphs, Commun. Comb. Optim. 2 (2017) 35-41.

Received 8 June 2017 Revised 19 October 2017 Accepted 23 October 2017 